\section{Zinc deficiency in children with Dengue viral infection}

\section{Lakkana Rerksuppaphol, ${ }^{1}$ Sanguansak Rerksuppaphol ${ }^{2}$}

1Department of Preventive Medicine, 2Department of Pediatrics, Faculty of Medicine, Srinakharinwirot University, Thailand

\begin{abstract}
Zinc deficiency is highly prevalent in low-income countries, with dramatic consequences to child health, in particular by impairing the immune system resulting in infection. This cross-sectional study aimed to determine the prevalence of zinc deficiency in Thai children who were admitted to hospital with a diagnosis of the dengue viral infection. Standard cut-off values according to age, sex, time of blood collection, and fasting status were used to define zinc levels. 32 patients were included in the analysis: The mean age was 7.3 years, of whom $56.3 \%$ were males. 11 (34.4\%) patients were diagnosed with dengue hemorrhagic fever and the remaining had dengue fever. The prevalence of zinc deficiency was $46.7 \%$, with boys having a higher risk of zinc deficiency than girls $(\mathrm{OR}=7.3$ : 95\%CI: 1.5-36.6). Fever duration and length of hospital stay were longer in children with zinc deficiency compared to those who had normal levels, albeit without a significant difference. The results of this study provide the rationale for larger studies that will better elucidate the relationship between zinc levels and the clinical outcomes of dengue disease.
\end{abstract}

\section{Introduction}

Zinc is a micronutrient that plays a pivotal role as a structural, catalytic, and signaling component within protein functions. About $10 \%$ of human proteins have the potential to bind with zinc, thus indicating that zinc is essential for numerous physiological processes. Zinc is important for growth and development, the immune system, the nervous system and endocrine functions, especially during infancy and childhood when the body is growing rapidly. ${ }^{1}$ Recommendations for zinc intake differ between males and females from the age of 15 years in most countries, but range from 2.9 to $10.0 \mathrm{mg} /$ day in children aged 5 years: $5.7-15.5 \mathrm{mg} /$ day for boys and $4.6-15 \mathrm{mg} /$ day for girls aged 10-15 years. ${ }^{2,3}$
Theoretically, half of the global population is at the risk of zinc deficiency due to low intake. Around 800,000 deaths among children below 5 years of age occur annually due to diarrhea $(176,000)$, pneumonia $(406,000)$, and malaria $(207,000) .4$ The high mortality rate among children resulting from these infections has been reported to be associated with inadequate zinc intake. 5 In developing countries, zinc deficiency is very common, and $61 \%$ of the population have low dietary zinc intake. Studies demonstrate that $49.4 \%$ of adolescent girls in Delhi suffer from zinc deficiency; 6 in Pakistan, $54.2 \%$ of children and $37.1 \%$ of preschool children are zinc-deficient; ${ }^{7}$ similarly, zinc deficiency is prevalent among various population groups in Sri Lanka, especially among preschool children and adolescents. ${ }^{8}$ In Thailand, the national prevalence of zinc deficiency is currently unknown; however, data relating to specific regions are available. The latest Thai national nutrition survey found the highest prevalence of stunting, a potential sign of zinc deficiency, in children in the Southern region; 9 based on serum zinc concentrations, $57 \%$ of school children are zinc deficient in North-East Thailand. 10

Zinc deficiency is associated with an impairment of the immune system. ${ }^{11,12}$ This may partially explain why these children are more prone to developing infections, including the dengue viral infection. Dengue disease is a leading cause of mortality in children younger than 15 years in South and South-East Asia. ${ }^{13}$ The World Health Organization (WHO) estimated that more than 2.5 billion people are at risk of dengue infection. ${ }^{13}$ The relationship between zinc levels and disease severity has been investigated, but current data are controversial. It has been shown that low serum zinc levels were significantly different in dengue infection with an increasing clinical severity, ${ }^{14}$ whereas another study found a significant difference in the number of lymphocytes in children with zinc deficiency to those with normal levels, without describing a correlation between zinc levels and disease severity. ${ }^{15}$ In Thai children with severe dengue disease and liver cell injury, low levels of zinc are reported, ${ }^{16}$ but the study did not specifically investigate the prevalence of zinc deficiency in this population.

This study aimed to determine the prevalence of zinc deficiency in Thai children with dengue disease. Standard cut-off values according to age, sex, time of blood collection, and fasting status were used to define zinc levels. ${ }^{17}$
Correspondence: Sanguansak Rerksuppaphol, Department of Pediatrics, Faculty of Medicine, Srinakhariwirot University, 62 Mo 7, RangsitNakorn Nayok Rd., Nakorn Nayok, 26120 Thailand.

Tel.: +6629058055- Fax:+6637395275.

E-mail: sanguansak_r@hotmail.com

Key words: dengue disease, zinc, children, zinc deficiency.

Acknowledgements: The authors would thank all children and their parents who participated in the study and the many people that assisted with the research project. The authors also thank Elisa Sala, $\mathrm{PhD}$ Medical Writer, for her medical editorial assistance.

Contributions: the authors contributed equally.

Conflict of interest: the authors declare no conflict of interest.

Funding: this study was supported by grants from Srinakharinwirot University, Thailand.

Received for publication: 28 August 2018. Revision received: 27 November 2018.

Accepted for publication: 5 December 2018.

This work is licensed under a Creative Commons Attribution NonCommercial 4.0 License (CC BY-NC 4.0).

C Copyright L. Rerksuppaphol and

S. Rerksuppaphol, 2019

Licensee PAGEPress, Italy

Pediatric Reports 2019; 11:7386

doi:10.4081/pr.2019.7386

\section{Materials and Methods}

\section{Study design and population}

From January to September 2016 a cross-sectional study was conducted in children admitted to the pediatric unit of MSMC Srinakharinwirot University Hospital with a diagnosis of dengue fever (DF) or dengue hemorrhagic fever (DHF), according to the modified World Health Organization (WHO) classification. ${ }^{13}$ Children who had acute febrile illness were included in the study if they presented with two or more of the following conditions: 1 ) nausea and vomiting; 2) rash; 3 ) aches and pain; 4) tourniquet test positive; 5) leukopenia (white blood cell count $\leq 5,000 / \mathrm{mm}^{3}$ ); 6) positive for any Dengue serology tests (Dengue IgG/IgM/NS1 antigen); 7) any warning signs (abdominal pain or tenderness, persistent vomiting, clinical fluid accumulation, mucosal bleeding, lethargy or restlessness, liver enlargement $>2 \mathrm{~cm}$, rising hematocrit (5-10\%) and thrombocy- 
topenia (platelet count $<150,000$ cells $/ \mathrm{mm}^{3}$ ). Children younger than 1 year or with chronic systemic diseases were excluded.

The study protocol was approved by the ethics committee of Srinakharinwirot University. Informed written consent was obtained from parents or legal guardians before enrolment. Parents and children could withdraw the consent at any time during the study.

\section{Measurements}

After enrolment, demographic characteristics and anthropometric data were collected by trained staff. Weight was measured in lightweight clothing to the nearest $0.1 \mathrm{~kg}$ using a digital scale. Height was measured to the nearest millimeter using a height rod. Body mass index (BMI) was calculated as the ratio of height $(\mathrm{kg})$ to square of height (m). Details on clinical history such as duration of fever, nausea or vomiting, aches and pain or bleeding were recorded. Clinical assessments were performed by attending physicians. The tourniquet test was performed by inflating the blood pressure cuff to a point midway between systolic and diastolic blood pressure and maintaining it for 5 minutes. Two minutes after cuff deflation, petechiae below the antecubital fossa were counted. A positive test was 10 or more petechiae per square inch. ${ }^{18}$

Blood samples were taken by venipuncture at the time of admission to assess the complete blood count (CBC), blood urea nitrogen, creatinine, electrolytes, dengue serology tests, serum albumin, aspartate transaminase, alanine transaminase, and serum zinc levels. A dengue serology test was performed by a lateral flow chromatographic immunoassay using a Dengue Combo Test kit (Encode $\AA$, Zhuhai, P.R.China). Serum zinc levels were measured by flame atomic absorption spectrometry. The time of blood sampling and the fasting status were recorded. Zinc deficiency was defined when serum zinc levels were below the lower cut-off, according to age, sex, fasting status, and time of blood sampling. Briefly, lower cut-offs for serum zinc levels were: 1 ) age $<10$ years: morning $65 \mathrm{mg} / \mathrm{dL}$, afternoon $57 \mathrm{mg} / \mathrm{dL}$; 2) male, age $\geq 10$ years: morning fasting $70 \mathrm{mg} / \mathrm{dL}$, morning non-fasting $66 \mathrm{mg} / \mathrm{dL}$, afternoon $59 \mathrm{mg} / \mathrm{dL}$; and 3 ) females, age $\geq 10$ years: morning fasting $74 \mathrm{mg} / \mathrm{dL}$, morning nonfasting $70 \mathrm{mg} / \mathrm{dL}$, afternoon $61 \mathrm{mg} / \mathrm{dL} \cdot{ }^{17}$

A diagnosis of DHF was done in patients who had signs of hemorrhagic manifestations, such as positive tourniquet test, petechiae, ecchymoses, purpura, or bleeding from mucosa, gastrointestinal tract or other locations, thrombocytopenia and evidence of plasma leakage such as rise of hematocrit $\geq 20 \%$ from baseline, pleural effusion or ascites. Other patients who had no signs of DHF were classified as DF. During the study, patients with signs of shock-pulse pressure $\leq 20 \mathrm{mmHg}$ with increased diastolic pressure or hypotension by age were diagnosed as having dengue shock syndrome (DSS). The choice of dengue treatment and decision of discharge were managed by attending physicians who were not involved in the study. Duration of defervescence, length of hospital stays, and clinical outcomes were recorded. Participants who had body temperature of $37.8^{\circ} \mathrm{C}$ or above were considered as having fever. Defervescence was defined as the first time at which body temperature fell to a normal level $\left(37.8^{\circ} \mathrm{C}\right.$ or below) for 2 consecutive measurements at 4-hour intervals. Clinical features, laboratory findings, and outcomes of treatment were compared between patients who had normal zinc levels and those with zinc deficiency.

The primary objective was to determine the prevalence of zinc deficiency in patients with dengue infection. The secondary objective was to assess the differences of clinical features and outcomes between DVI patients who had normal zinc levels or those with zinc deficiency. We estimated that the prevalence of zinc deficiency in dengue infection was $75 \% 15$ with an allowable error of $15 \%$. A sample size of 32 patients was required to detect a significant level of 0.05 .

\section{Statistical analysis}

Normal distribution of continuous data was assessed using a one-sample Kolmogorov-Smirnov test. Normally distributed variables were reported as mean and standard deviation, whereas non-normally distributed variables were described as median and interquartile range (IQR). Pearson's chi-square test or Fisher's exact test were used to compare proportions between groups, as appropriate. The Student's $t$-test and the Mann-Whitney U test were employed to determine the differences between normally distributed and non-normally distributed variables, respectively. A logistic regression analysis was performed to estimate the odds ratio (OR) and $95 \%$ confidence interval $(95 \% \mathrm{CI})$ of the likelihood of zinc deficiency. A twosided P-value of less than 0.05 was considered statistically significant. Statistical analysis was performed using SPSS version 23.0 statistical package (SPSS, Chicago, IL, USA).

[Pediatric Reports 2019; 11:7386]

\section{Results}

33 children admitted to hospital with a diagnosis of DH/DHF were invited to participate at the study: one patient declined to participate, while 32 patients agreed and were included. $18(56.3 \%)$ patients were boys with a mean age of 7.3 years (SD. 3.4 years). The median duration of fever before admission was 3.0 days (range 1-7 days).

Nausea and vomiting were reported in 27 patients $(84.8 \%)$, rash in $5(15.6 \%)$, headache in $24(35.0 \%)$, and myalgia in 10 $(31.3 \%) .7$ patients $(21.9 \%)$ had a positive result in the tourniquet test, whereas 3 (9.4\%) patients had gastrointestinal bleeding. $11(34.4 \%)$ patients were diagnosed with DHF; the remaining had DF.

None of the patients had DSS at the time of admission or during the treatment. Clinical manifestations of DVI are shown in Figure 1. Laboratory findings revealed that 8 patients $(25.0 \%)$ had leukopenia and 26 $(81.3 \%)$ had thrombocytopenia. 7 patients $(37.5 \%)$ had high aspartate aminotransferase levels, whereas $3(9.4 \%)$ had high alanine aminotransferase levels.

The mean serum zinc level upon admission was $69.1 \mathrm{mg} / \mathrm{dL}$ and 15 patients (46.9\%) had zinc deficiency. Patients who had zinc deficiency had average serum zinc levels of $53.3 \mathrm{mg} / \mathrm{dL}$, compared to an average of $83.0 \mathrm{mg} / \mathrm{dL}$ in the patients with normal serum zinc levels. Boys had a higher risk of having zinc deficiency than girls $(\mathrm{OR}=7.3 ; 95 \% \mathrm{CI}: 1.5-36.6)$, without other significant differences in terms of age, weight, height, BMI, clinical characteristics or basic laboratory parameters at the time of hospitalization (Table 1). Patients with zinc deficiency had a longer duration of fever compared to patients with normal zinc levels $(5.0 \pm 2.1$ vs $3.9 \pm 2.4$ days, respectively; $\mathrm{P}=0.190$ ). The length of hospital stay was slightly shorter for patients with normal zinc levels, without a statistically significant difference. During hospitalization, none of the patient showed signs of severe hemorrhage or plasma leakage, such as pleural effusion, ascites, or hypovolemic shock.

\section{Discussion}

This cross-sectional study reported that the prevalence of zinc deficiency in children with dengue disease was $46.7 \%$ at the time of admission; boys seem to have a higher risk of zinc deficiency than girls, regardless of age, weight, height, and clinical characteristics. Patients with zinc deficiency had slightly longer duration of fever 
and hospital staying than did those with normal zinc levels.

The prevalence of zinc deficiency in children with dengue disease described here was lower to that reported for the general population of school children in North-East Thailand $(57 \%)^{10}$ and as described in previous studies that specifically investigated children with dengue disease. Widago et al reported low serum zinc levels in 34 children out of $45(75.5 \%)$, using a cut-off of $9.18 \mu \mathrm{mol} / \mathrm{L} ; 15$ Yuliana et al. reported a prevalence of $78.3 \%$, using a cut-off of 70$90 \mu \mathrm{g} / \mathrm{dL}$, with most children in their sample being younger than 10 years. ${ }^{14}$ The use of different cut-off values makes it difficult to compare between studies. It should be noted that children who we considered as having normal zinc levels had a mean zinc concentration of $83.3 \mu \mathrm{g} / \mathrm{dL}$, a value below the cut-off used by Yuliana et al. ${ }^{14}$ The use of standard cut-off criteria to define zinc deficiency ${ }^{17}$ is also advisable to make possible a comparison between studies.

In our study, we decided to use cut-off values that kept into account possible confounding factors, such as age, sex, time of day of blood sampling, and fasting status, to assess an accurate estimate of zinc deficiency. During childhood, males have lower serum zinc concentrations than females, while in late childhood (at about 10 years) zinc levels in boys begin to exceed those of girls; age is significantly associated with serum zinc concentration $(\mathrm{P}<0.0001)$ and serum zinc concentrations are lowest in young children, and increases steadily with age. The time of day of blood sampling has a significant effect on serum zinc concentrations $(\mathrm{P}<0.0001)$, such that serum zinc is higher in the morning samples than in the afternoon or evening samples. The fasting status is more relevant in elderly subjects. ${ }^{17}$ As expected, we found that the prevalence of zinc deficiency was higher in boys than in girls.

We did not observe any difference in terms of disease severity and clinical outcomes in children with zinc deficiency or with normal zinc levels, since the primary aim of this study was not to investigate this point and the sample size was not adequate to estimate the difference. However, longer durations of fever and hospital stay were

Table 1. Clinical and laboratory characteristics in children with dengue viral infection with normal zinc levels or zinc deficiency.

\begin{tabular}{|c|c|c|c|}
\hline & $\begin{array}{l}\text { Patients with normal zinc levels } \\
\qquad(\mathrm{n}=17)\end{array}$ & $\begin{array}{l}\text { Patients with zinc deficiency } \\
\qquad(\mathrm{n}=15)\end{array}$ & P-value \\
\hline Boys; n (\%) & $6(35.3)$ & $12(80.0)$ & 0.016 \\
\hline Age (yr) & 7.23 .9 & 7.32 .8 & 0.919 \\
\hline Weight (kg) & $29.1(18.3)$ & $21.5(6.2)$ & 0.126 \\
\hline Height $(\mathrm{cm})$ & 123.926 .1 & 120.412 .4 & 0.628 \\
\hline $\begin{array}{l}\text { Body mass index }\left(\mathrm{kg} / \mathrm{m}^{2}\right) \\
\text { Clinical features, } \mathrm{n}(\%) \\
\text { Body temperature }(\mathrm{oC}) \\
\text { Duration of fever before admission (days) } \\
\text { Duration of fever during admission (days) } \\
\text { Total duration of fever (days) } \\
\text { Nausea or vomiting } \\
\text { Abdominal pain } \\
\text { Headache } \\
\text { Myalgia/arthralgia } \\
\text { Hepatomegaly } \\
\text { Positive tourniquet test } \\
\text { Petechial haemorrhage } \\
\text { Epistaxis } \\
\text { Gastrointestinal bleeding }\end{array}$ & $\begin{array}{c}17.04 .8 \\
38.40 .7 \\
2.72 .0 \\
1.21 .3 \\
3.92 .4 \\
15(88.2) \\
9(52.9) \\
13(76.5) \\
6(35.3) \\
3(17.6) \\
4(23.5) \\
4(23.5) \\
0(0) \\
1(5.9)\end{array}$ & $\begin{array}{c}14.62 .5 \\
38.50 .9 \\
3.31 .8 \\
1.71 .3 \\
5.02 .1 \\
12(80.0) \\
9(60.0) \\
11(73.3) \\
4(26.7) \\
4(26.7) \\
3(20.0) \\
1(6.7) \\
3(20.0) \\
2(13.3)\end{array}$ & $\begin{array}{l}0.085 \\
0.821 \\
0.407 \\
0.280 \\
0.190 \\
0.645 \\
0.735 \\
1.000 \\
0.712 \\
0.678 \\
1.000 \\
0.338 \\
0.092 \\
0.589\end{array}$ \\
\hline $\begin{array}{l}\text { Dengue classification, } \mathrm{n}(\%) \\
\text { Dengue fever } \\
\text { Dengue haemorrhagic fever }\end{array}$ & $\begin{array}{l}11(64.7) \\
6(35.3)\end{array}$ & $\begin{array}{l}10(66.7) \\
5(33.3)\end{array}$ & 1.000 \\
\hline Length of hospital stay (days) & 2.81 .5 & 3.61 .4 & 0.141 \\
\hline $\begin{array}{l}\text { Laboratory findings } \\
\text { Hemoglobin }(\mathrm{g} / \mathrm{dL}) \\
\text { Hematocrit }(\%) \\
\text { Leukocyte count }\left(\times 103 / \mathrm{mm}^{3}\right) \\
\text { Platelets }\left(\mathrm{x} 10^{3} / \mathrm{mm}^{3}\right) \\
\text { Albumin }(\mathrm{g} / \mathrm{dL}) \\
\text { Aspartate aminotransferase* (U/L) } \\
\text { Alanine aminotransferase* }(\mathrm{U} / \mathrm{L}) \\
\text { Zinc }(\mathrm{g} / \mathrm{dL}) \\
\text { Sodium }(\mathrm{mmol} / \mathrm{L}) \\
\text { Potassium }(\mathrm{mmol} / \mathrm{L}) \\
\text { Chloride }(\mathrm{mmo} / \mathrm{L}) \\
\text { Bicarbonate }(\mathrm{mmol} / \mathrm{L}) \\
\text { Blood urea nitrogen }(\mathrm{mg} / \mathrm{dL}) \\
\text { Creatinine }(\mathrm{m} / \mathrm{dL})\end{array}$ & $\begin{array}{c}12.51 .6 \\
38.04 .9 \\
6.83 .3 \\
112.436 .9 \\
4.10 .5 \\
30.0(20.5) \\
17.0(7.5) \\
83.023 .5 \\
135.03 .2 \\
4.00 .4 \\
104.53 .0 \\
18.73 .0 \\
10.53 .4 \\
0.40 .1\end{array}$ & $\begin{array}{c}12.21 .1 \\
36.33 .1 \\
8.33 .5 \\
109.529 .8 \\
3.90 .3 \\
35.0(48.0) \\
20.0(18.0) \\
53.39 .0 \\
134.31 .7 \\
3.90 .3 \\
104.32 .1 \\
20.93 .1 \\
10.55 .5 \\
0.40 .2\end{array}$ & $\begin{array}{l}0.541 \\
0.241 \\
0.231 \\
0.807 \\
0.060 \\
0.911 \\
0.628 \\
<0.001 \\
0.483 \\
0.538 \\
0.826 \\
0.055 \\
0.984 \\
0.987\end{array}$ \\
\hline
\end{tabular}

*Presented as Median (IQR) 


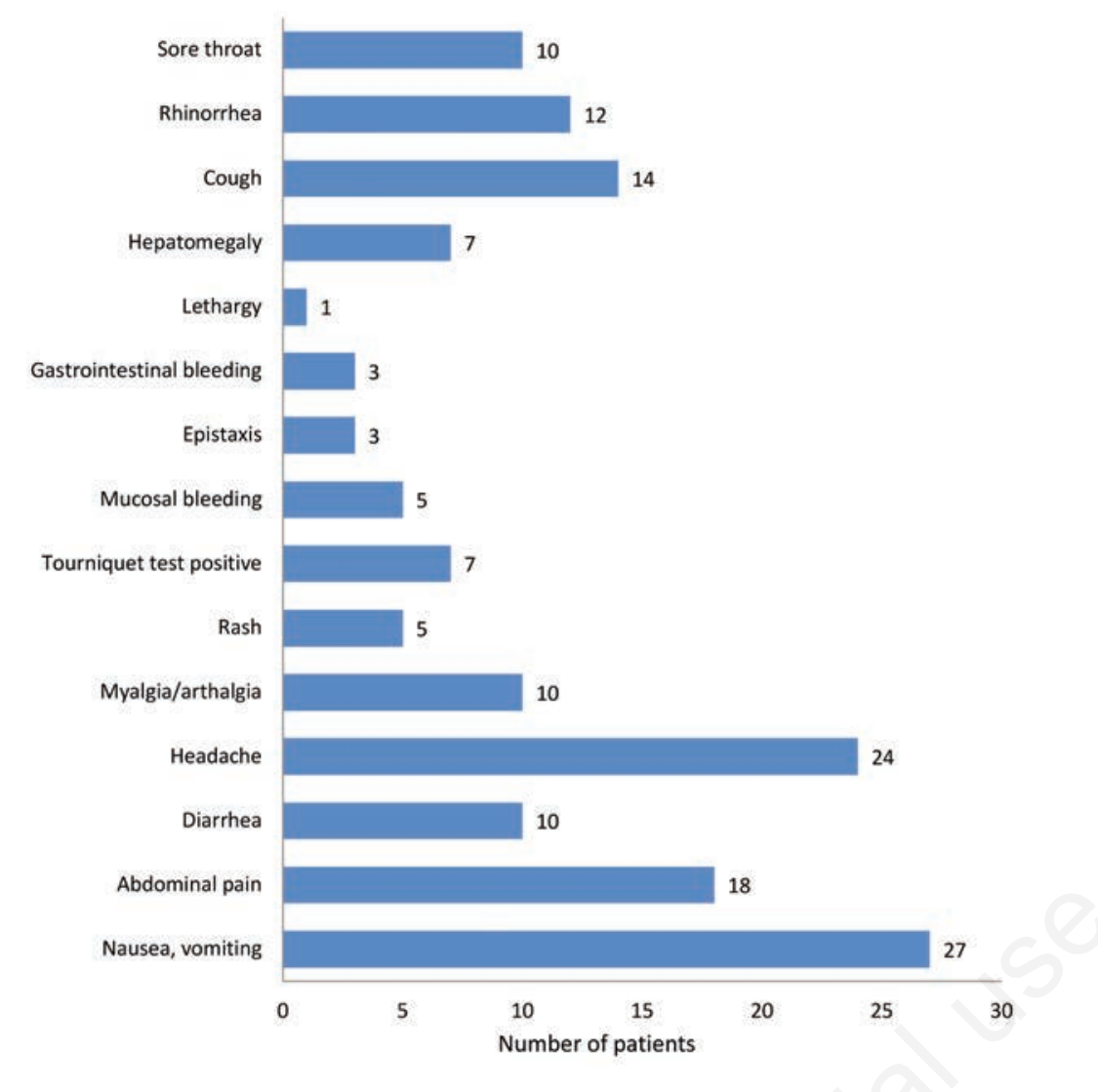

Figure 1. Clinical manifestations of dengue viral infection.

reported in children with zinc deficiency, suggesting that these children had a worse overall health status that affected the time of defervescence and recovery than those without zinc deficiency.

Data about zinc supplementation in children with dengue disease are not available yet, but WHO guidelines recommend oral zinc for the clinical management of childhood diarrhea; 19 therefore, the use of oral zinc supplementation in children with dengue disease and diarrhea or who need hydration may be appropriate. Furthermore, zinc plays a significant role in the maintenance of normal immunological functions; therefore, measures for zinc supplementation should be taken in regions where zinc deficiency and dengue infection are endemic. The study has some limitations. Firstly, the population size was not adequate enough to determine a possible correlation between zinc deficiency and clinical outcomes of dengue disease; further studies are recommended to assess the impact of zinc deficiency in the clinical outcomes of dengue disease and to evaluate zinc supplementation as preventive or adjuvant treatment for this disease. Secondly, the duration of hospital stay was dependent on the deci- sion to discharge by attending clinicians and was not based on a priori established criteria. Thirdly, information about the duration of fever before the admission was selfreported by patients or their guardians and not may be wholly accurate. Fourthly, during the study, we did not measure the zinc intake from food. All these aspects may prevent precise determination of the relationship between clinical outcomes and zinc deficiency, but exactly mirror the real-world clinical practice in Thailand. Finally, no patients with DSS were included in the analysis.

\section{Conclusions}

Zinc deficiency in Thai children with dengue disease is highly prevalent and patients with low zinc levels show slightly longer duration of fever and length of hospital stay than children with basal normal levels. The use of standard cut-off criteria is valuable to better identify children at risk of zinc deficiency and, eventually, perform ad $h o c$ intervention in the form of zinc supplementation.

\section{References}

1. Kambe T, Fukue K, Ishida R, et al. Overview of Inherited Zinc Deficiency in Infants and Children. J Nutr Sci Vitaminol (Tokyo) 2015;61:S44-6.

2. Moran VH, Stammers AL, Medina MW, et al. The relationship between zinc intake and serum/plasma zinc concentration in children: a systematic review and dose-response meta-analysis. Nutrients 2012;4:841-58.

3. Iglesia I, Doets EL, Bel-Serrat S, et al. Physiological and public health basis for assessing micronutrient requirements in children and adolescents. The EURRECA network. Matern Child Nutr 2010;6:84-99.

4. Brown K, Wuehler S, Peerson J. The importance of zinc in human nutrition and estimation of the global prevalence of zinc deficiency. Food Nutr Bull 2001;22:113-25.

5. Penny ME. Zinc supplementation in public health. Ann Nutr Metab 2013;62:31-42.

6. Kapil U, Toteja GS, Rao S, et al. Zinc deficiency amongst adolescents in Delhi. Indian Pediatr 2011;48:981-2.

7. Ejaz MS, Latif N. Stunting and micronutrient deficiencies in malnourished children. J Pak Med Assoc 2010;60:543-7.

8. Hettiarachchi M, Liyanage C. Coexisting micronutrient deficiencies among Sri Lankan pre-school children: a community-based study. Matern Child Nutr 2012;8:259-66.

9. Nutrition Division, Ministry of Public Health. The fifth national nutrition survery: The Operation of Thai Nutritional's Program 2005. Nonthaburi (Thailand): Ministry of Public Health; 2005.

10. Thurlow RA, Winichagoon P, Pongcharoen $\mathrm{T}$, et al. Risk of zinc, iodine and other micronutrient deficiencies among school children in North East Thailand. Eur J Clin Nutr 2006;60:623-32.

11. Black RE. Therapeutic and preventive effects of zinc on serious childhood infectious diseases in developing countries. Am J Clin Nutr 1998;68:476S-9S.

12. Basnet S, Mathisen M, Strand TA. Oral zinc and common childhood infections-An update. J Trace Elem Med Biol 2015;31:163-6.

13. World Health Organization. Dengue guidelines for diagnosis, treatment, prevention and control. Geneva: WHO press, 2009.

14. Yuliana N, Fadil R, Chairulfatah 
A.Serum zinc levels and clinical severity of dengue infection in children. Paediatri Indones 2009;49:309-14.

15. Widagdo. Blood zinc levels and clinical severity of dengue hemorrhagic fever in children. Southeast Asian J Trop Med Public Health 2008;39:610-6.

16. Laoprasopwattana

K, Tangcheewawatthanakul C, Tunyapanit $\mathrm{W}$, et al. Is zinc concentration in toxic phase plasma related to dengue severity and level of transaminases? PLoS Negl Trop Dis 2013;7:e2287.

17. Hotz C, Peerson JM, Brown KH. Suggested lower cutoffs of serum zinc concentrations for assessing zinc status: reanalysis of the second National Health and Nutrition Examination Survey data (1976-1980). Am J Clin Nutr 2003;78:756-64.
18. Centers for Disease Control and Prevention. Touniquet test. [cited 2017 July 10]. Available from: https:/www.cdc.gov/dengue/training/c $\mathrm{me} / \mathrm{ccm} /$ Tourniquet$\% 20$ Test_F.pdf.

19. Lazzerini M, Wanzira H. Oral zinc for treating diarrhoea in children. Cochrane Database Syst Rev 2016;12:CD005436 